\title{
EFFECTS OF A WATER HAMMER AND CAVITATION ON VIBRATION TRANSIENTS IN A RESERVOIR-PIPE-VALVE SYSTEM
}

\author{
YANG MiaO \\ Faculty of Materials and Manufacturing, Beijing University of Technology, Beijing, China, and \\ Beijing Key Laboratory of Advanced Manufacturing Technology, Beijing University of Technology, Beijing, China \\ Zaihui Qiu, Xiaolu Zhang, Yuncheng Jiang \\ Faculty of Materials and Manufacturing, Beijing University of Technology, Beijing, China \\ Jun PAN, Yi LiU \\ Nanjing Electromechanical Hydraulic Engineering Center \\ Li ZHANG, Kun Li \\ China Nuclear Power Technology Research Institute Co., Ltd. \\ e-mail: likun0755@hotmail.com
}

\begin{abstract}
An extraordinary phenomenon with violent oscillation, accompanied by an abnormal "click" sound is observed during the valve closing in a fuel feeding pipe system. A fluctuation model with flow cavitation, in which time-varying stiffness, time-varying damping coefficients and flow cavitation are comprehensively considered, is proposed. On this basis, a dynamic vibration equation is established and an expression of flow pressure at the valve port is derived. The critical displacement of the flow cavitation system is defined. When the vibration amplitude reaches the critical displacement, cavitation occurs. Based on this model, simulation of vibration displacement and flow pressure is given. The simulated transient process shows the phenomena of vibration fluctuation with cavitation. The results are compared with the experimental data measured by a pressure sensor. Under our experimental conditions, the critical displacement of vibration is $1.41 \mathrm{~mm}$, and the average relative pressure peak error is 0.022. The comparison between the simulation and experimental results shows that they are in an acceptable agreement.
\end{abstract}

Keywords: reservoir-pipe-valve system (RPV system), water hammer, vibration, cavitation

\section{Introduction}

In the reservoir pipeline valve (RPV) system, water hammer (WH) will occur when the valve is closed, which may affect stability of the system or even may cause unexpected damage. A simplest model is that the structure of the system is regarded as a rigid body, the fluid in the pipe is an elastic body, and WH effect produces elastic waves in the pipe. In general, partial differential equations (Wang and Eat Tan, 1997; Tijsseling, 2003; Yang et al., 2004) related to momentum and continuity are used to describe the pipe flow, including the fluid solid interaction (Wiggert and Tijsseiling, 2001; Keramat et al., 2012; Henclik, 2015; Ferras et al., 2018) and the influence of the pipe support (Covas et al., 2005; Zanganeh et al., 2015; Adamkowski et al., 2017; Henclik, 2018b). In conclusion, the flow field is related to an unsteady pipe flow, especially in the case of fast transient, the time variation of wall friction must be considered. Zarzycki and Urbanowicz reported a series of important works on the unsteady pipe flow. They proposed a simple method to determine coefficients of the weight function (Zarzycki et al., 2011; Urbanowicz, 2017a), approximated the weight function to the sum of exponential components (Urbanowicz and Zarzycki, 2012), and improved the lumped friction model for estimating the basic parameters 
(Urbanowicz and Zarzycki, 2015). They also simulated transients of the turbulent pipe flow (Zarzycki et al., 2007). In their work, a time-varying drag model was used to simulate transient phenomena of the unsteady flow.

The instantaneous wall shear stress is the sum of two parts, one is related to the quasi steady state, the other is related to the unsteady state. The time-varying component of the stress is derived as convolution of the local velocity variation of the liquid and the weighting function. It is known that for the unsteady liquid flow in pipes, when pressure drops to saturated vapor pressure, flow cavitation will be formed. For the unsteady liquid flow with cavitation, the column separation model was established earlier (Streeter, 1969; Wylie and Streeter, 1978). Zarzycki and Urbanowicz studied the calculation model and method of the weight function (Urbanowicz and Zarzycki, 2008; Urbanowicz et al., 2012) and the influence of hydraulic pipe parameters (Zarzycki and Urbanowicz, 2008) on the cavitation unsteady flow. Urbanowicz (2017b) systematically investigated time-varying hydraulic resistance. In his work, wall shear stress was expressed as the sum of a quasi-steady and time-varying components, and the calculating was completed with a simplified method.

It is observed that when the feed flow rate reaches a certain value, the feed pipe system vibrates violently when the valve is closed, accompanied by an abnormal "click" sound. In the paper (Miao et al., 2020), we proposed a fluctuation model to simulate the vibration process of the system. The simulation successfully explained the occurrence of the violent random vibration and abnormal sound in the experiment. However, the calculation results also have a serious defect, e.g. some negative pressure values appear in the simulations. Obviously, these pressure values are unreasonable and unrealistic. In order to improve the simulation calculation, a model of fluctuation with flow cavitation is proposed in this paper. In the model, time-varying coefficients of the stiffness and damping as well as flow cavitation are taken into account. A dynamic vibration equation is established, and the expression of flow pressure derived. A critical displacement of vibration is introduced, which means that flow cavitation will take place if the vibration amplitude reaches this value. On this basis, the simulation process of vibration displacement and flow pressure is given. Under our experimental conditions, the critical displacement of vibration is $1.41 \mathrm{~mm}$ and the average relative pressure peak error is 0.022 . The comparison between the simulation results and the experimental data shows that they are in an acceptable agreement.

\section{Theoretical model}

\subsection{Physical model of the RPV system}

A scheme of the RPV system (top in Fig. 1) can be classified as two parts. The first consists of a fuel delivery pump (1), filter (2), throttle valve (3), safety valve (4), pressure transducer (5), switch valve (6) and a flowmeter (7). The second includes a part of the fuel transfer pipeline, a directional valve (8) which control the fuel flow to consumption unit (9) or to a low pressure reservoir. The pipeline, fixed on the base with irregular spaced supports, is made of aluminum alloy, and its length, diameter and thickness are $8.7 \mathrm{~m}, 30 \mathrm{~mm}$ and $3 \mathrm{~mm}$, respectively. The switch valve (FHH-2) can be closed within $10 \mathrm{~ms}$. The pressure transducer (MEAS U5300) is mounted at $200 \mathrm{~mm}$ from the switch valve.

The middle and bottom schemes in Fig. 1 show the physical model of the RPV system in which the cavitation phenomenon occurs. The output of the delivery pump is taken as the starting point, that is $x=0$, the position of the valve is at $x=L$, and the flow rate of the fuel in pipeline is $v(x, t)$. In order to study the influence of $\mathrm{WH}$ on the vibration of the RPV system, the support with irregular distribution is simplified as a spring damper connected with the valve section. Let $\alpha$ and $\beta$ denote the stiffness and damping coefficients, respectively. Two hypotheses are put forward: 

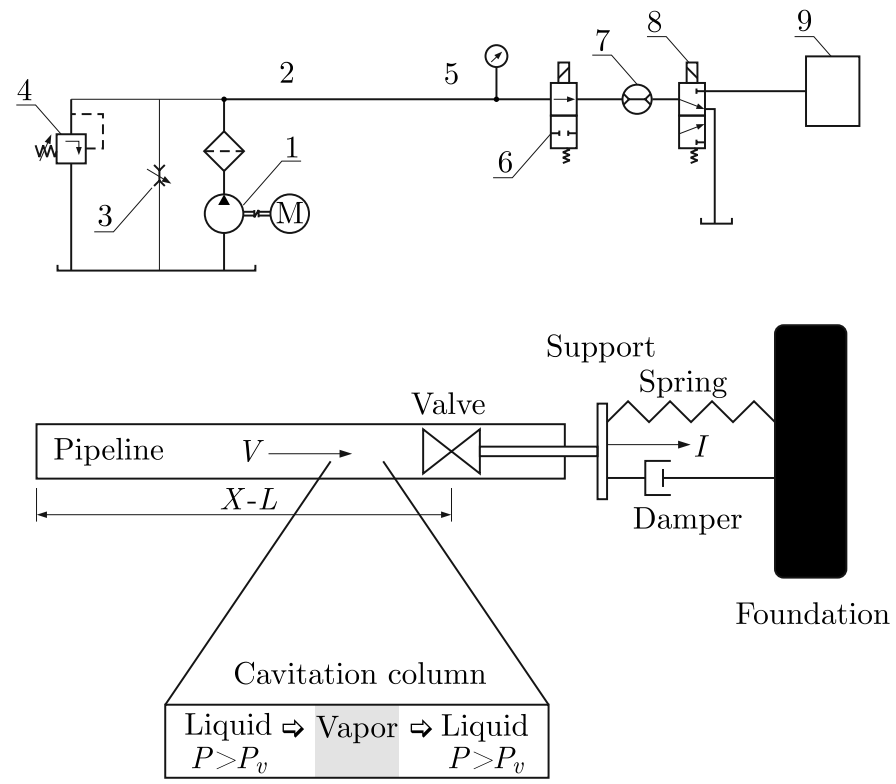

Fig. 1. Physical model of the RPV system

(i) For severe vibration, the stiffness and damping coefficients are time-varying. They can be expressed as $\alpha_{0}+\Delta \alpha(t)$ and $\beta_{0}+\Delta \beta(t), \alpha_{0}$ and $\beta_{0}$ are constants, such as the stiffness and damping coefficients in classical theory. Time-varying $\Delta \alpha(t)$ and $\Delta \beta(t)$ are used to describe the fluctuation of the system.

(ii) When the pressure near the valve drops below the saturated vapor pressure, a flow cavitation column will appear. The pressure in the cavitation zone is equal to the vapor pressure $P_{v}$. On both sides of the cavitation column, the liquid pressure is equal to the vapor pressure, i.e. $P=P_{v}$.

\subsection{Mathematical model of the pipeline flow}

One-dimensional unsteady flow in pipes is often described by continuity and momentum equations as well as boundary and initial conditions (Wylie and Streeter, 1978):

- continuity equation

$$
\frac{\partial P}{\partial t}+v \frac{\partial P}{\partial x}+\rho c^{2} \frac{\partial v}{\partial x}=0
$$

- momentum equation

$$
\frac{\partial v}{\partial t}+v \frac{\partial v}{\partial x}+\frac{1}{\rho} \frac{\partial P}{\partial x}+g \sin \gamma+\frac{4 \tau_{s}}{\rho D}=0
$$

where $x$ is the positional variable along the pipe axis, $c$ is the pressure wave speed, $t$ is time, $v$ is the instantaneous mean flow velocity, $P$ is pressure, $\rho$ is fluid density, $\tau_{s}$ is wall shear stress, $g$ is gravitational acceleration, $\gamma$ is pipe slope angle and $D$ is the inner diameter of the pipe.

\subsection{Vibration model of the RPV system}

When the valve is closed, the pressure pulsation of water hammer acts on the pipeline system. The energy exchange between the flow and structure causes vibration. According to the physical model shown in Fig. 1, the system can be simplified as a harmonic oscillator at the valve node. The vibration of the pipeline system is essentially caused by the elastic recovery, damping and 
water hammer force acting on the valve node. Therefore, in the case of fluctuation vibration, the dynamic equation of pipeline system is

$$
m \ddot{y}+\left[\beta_{0}+\Delta \beta(t)\right] \dot{y}+\left(\alpha_{0}+\Delta \alpha\right) y=P S
$$

where $m$ is mass of the pipe system, $\alpha_{0}+\Delta \alpha(t)$ and $\beta_{0}+\Delta \beta(t)$ are time varying stiffness and damping coefficients, $y$ is the expansion length of the spring which is equal to the displacement of the valve, $\dot{y}$ is the valve velocity, $S$ is the cross-sectional area, $P$ is the instantaneous flow pressure.

It should be pointed out that when the vibration amplitude of the system reaches a certain degree, the flow cavitation will occur in the pipeline and form a cavitation column. When the cavitation column acts on the valve, the system still oscillates, and the vibration satisfies dynamic equation (2.3). But the pressure in the equation should be an instantaneous vapor pressure, and the coefficient changing with time can be regarded as a constant. When $\mathrm{WH}$ pressure changes to the vapor pressure, the vibration process of the pipeline system is continuous.

\section{Analysis of the system parameters}

\subsection{Boundary and initial conditions}

\subsubsection{Initial conditions}

For the initial steady flow and oscillator, we have the initial position and velocity of the valve as $\left.y(t)\right|_{t=0}=y_{0}$ and $\left.\dot{y}(t)\right|_{t=0}=0$. In practical, the initial flow rate $\left.v(x, t)\right|_{t=0}$ and the initial pressure $\left.P(x, t)\right|_{t=0}$ change along the pipeline. In this paper, in order to solve dynamic equation (2.3), we only consider the values at the valve section. For short pipe lengths and to simplify calculation, they are considered as constants and marked as $v_{0}$ and $P_{0}$ before the valve closing. In the case of the initial flowrate $v_{0}$, the kinetic energy is $m_{w} v_{0}^{2} / 2$, where $m_{w}$ is mass of the fuel in the pipeline. When the valve moves to the position $y_{0}$, the potential energy of the system is $\alpha y_{0}^{2} / 2$. According to the law of energy conservation, we obtain

$$
y_{0}=\sqrt{\frac{m_{w}}{\alpha}} v_{0}
$$

Thus, the value of the position $y_{0}$ can be obtained according to this equation (energy dissipation in motion is neglected here).

\subsubsection{Boundary conditions}

The boundary conditions at the left side of the pipeline $(x=0)$ are $P=$ const and $\partial v(x, t) / \partial x=0$ because the pulsation of the pump output is small.

The boundary condition at the right side of the pipeline $(x=L)$ is $v(L, t)=\dot{y}$ where $\dot{y}$ is the valve motion velocity. This means that the liquid and the valve vibrate at the valve node synchronously, i.e. the fluid velocity at the right end of the pipe is the same as that of the valve node. Because it is observed that the whole vibration of the RPV system takes place in the experiment and the pipeline is assumed as rigid, the distance between the delivery pump and the valve is still $x=L$.

\subsection{The excitation force and vibration dynamic equation}

When the valve starts closing, the relative velocity of the fluid is $v_{0}-v(x, t)$. According to the Joukovsky formula, the excitation force on the valve caused by $\mathrm{WH}$ is

$$
P S=\rho c\left(v_{0}-v\right) S
$$


According to the boundary condition, one knows

$$
v=v(L, t)=\dot{y}(t)
$$

Substituting equation (3.3) into equation (2.3), we obtain dynamic equations of the pipeline system vibration as

$$
m \ddot{y}+\left(\beta_{0}+\Delta \beta(t)+\rho c S\right) \dot{y}+\left(\alpha_{0}+\Delta \alpha\right) y=\rho c v_{0} S
$$

\subsection{Pressure in the pipeline}

The simulations of the WH effect in a one-dimensional conduit with cavitation were conducted by the column separation model (CSM) in the cases of considering both quasi-steady and unsteady friction losses (Urbanowicz and Zarzycki, 2008; Urbanowicz et al., 2012). In their works, equations (2.1) and (2.2) were solved using the method of characteristics (MOC). They acquired detailed changes of pressure in a pipe cross section near the valve. In this work, we assume that the fluid and valve are vibrating synchronously at the valve node, and try to establish a relationship between valve vibration and fluid pressure based on vibration dynamic equation (2.3). Suppose the fluid is impressible, and according to continuity equation (2.1), the relation between the velocity and the pressure in the pipeline can be expressed as

$$
\frac{\partial v(x, t)}{\partial x}+\frac{1}{\rho c^{2}} \frac{\partial P(x, t)}{\partial t}=0
$$

Then, with integrating equation (3.5), we have

$$
P(x, t)=P(x, 0)-\rho c^{2} \int_{0}^{t} \frac{\partial v(x, t)}{\partial x} d t
$$

where $P(x, 0)=P_{0}$, the initial pressure of the fluid in the pipeline.

Based on equations (2.1) and (2.2), the velocity function $v(x, t)$ in the pipeline can be expressed as a Fourier series (Henclik, 2018a) in the case of friction stress neglected, where the $n$-th order component is

$$
v_{n}(x, t)=v_{0 n} \cos \left(k_{n} x\right) \cos \left(\omega_{n} t\right)
$$

and $\omega_{n}=k_{n} c$. For simplicity, we only consider the fundamental component in the calculation.

It is assumed that the fluid velocity $v(x, t)$ at the valve input is equal to the valve node vibration velocity $\dot{y}$. Then, according to equation (3.7), we have

$$
\frac{\partial v(x, t)}{\partial x}=k \tan (k L) \dot{y}(t)
$$

where $k=\omega / c, c$ is the pressure wave speed and $\omega$ is the vibration angular frequency. In the case of non-fluctuation, $\omega=\sqrt{\alpha_{0} / m}$, and then

$$
k=\frac{1}{c} \sqrt{\frac{\alpha_{0}}{m}}
$$

Substituting equations (3.8) into equation (3.6), we obtain the instantaneous pressure in the pipeline $P(t)$ as

$$
P(t)=P(0)+\rho c \sqrt{\frac{\alpha_{0}}{m}} \tan (k L)\left[y_{0}-y(t)\right]
$$




\subsection{Critical displacement}

According to the assumption of cavitation, when the pressure in the valve section falls below the saturated vapor pressure, cavitation will appear. At the both sides of the cavitation column, the liquid pressure is equal to the vapor pressure $P_{v}$. Suppose that the liquid pressure $P(t)$ is equal to the saturated vapor pressure at a moment, then according to the equation (3.10), the corresponding displacement can be written as

$$
y_{c}=y_{0}+\frac{P(0)-P_{v}}{\rho c \tan (k L)} \sqrt{\frac{m}{\alpha_{0}}}
$$

The displacement $y_{c}$ is defined as the critical displacement, and the criterion of flow cavitation is given. When the vibration amplitude is within the critical displacement range, there is no flow cavitation phenomenon, and the exciting force is caused by WH pressure. However, when the vibration amplitude reaches the critical displacement, a vapor area will appear near the valve. In the case of cavitation, the vapor is usually set as a constant value although its pressure has very small changes during the system vibration.

Physically, the pipeline system vibrates under pressure pulsation of water hammer, and dynamic equation (3.4) is satisfied when the valve is closed. When the vibration displacement reaches the critical value, liquid cavitation occurs near the valve and a vapor column is formed. In this case, the pressure pulsation acting on the valve should be the vapor. Since the vapor pressure is far less than WH pressure, the vibration amplitude caused by the vapor alone is very small, which can be easily expressed as

$$
A_{0 v}=\frac{P_{v} S}{\alpha_{0}}
$$

In the case of cavitation and during the action of the vapor column, the system still vibrates and the vibration still conforms to dynamic equation (3.4). However, the liquid density $\rho$ in equation (3.4) should be replaced by the vapor density $\rho_{v}$. Meanwhile, the time-varying coefficients $\Delta \alpha(t)$ and $\Delta \beta(t)$ can be approximately considered as zero. No matter if under the action of water hammer or vapor pressure, the system vibrates. At the moment of pressure transition between the vapor and liquid, the system vibration is continuous.

\section{Results and discussion}

In the numerical calculation, the parameters of the line were as follows: pipe length $L=8.7 \mathrm{~m}$, inner diameter $D=30 \mathrm{~mm}$, thickness $e=3 \mathrm{~mm}$, density $\rho_{s}=2.73 \cdot 10^{3} \mathrm{~kg} / \mathrm{m}^{3}$. The working liquid is kerosene RP-5 with density $\rho=0.78 \cdot 10^{3} \mathrm{~kg} / \mathrm{m}^{3}$. The vapor density $\rho_{v}=0.87 \mathrm{~kg} / \mathrm{m}^{3}$ and its saturated vapor pressure $P_{v}=5.1 \mathrm{kPa}$. The wave speed $c=1324 \mathrm{~m} / \mathrm{s}$, mass $m=9.3 \mathrm{~kg}$ estimated as the sum of masses of the fluid, pipe and the valve. The initial flow velocity is $0.8 \mathrm{~m} / \mathrm{s}$. The stiffness and damping coefficients are $\alpha_{0}=274 \cdot 10^{2} \mathrm{~N} / \mathrm{mm}$ and $\beta_{0}=54 \mathrm{~N} \mathrm{~s} / \mathrm{m}$. The values of stiffness and damping coefficients are estimated with the formula $\alpha_{0}=4 \pi^{2}[c /(4 L)]^{2} m_{w}$ and $\beta_{0}=2 \xi \sqrt{K m_{w}}$, where $m_{w}$ is mass and $\xi$ is the non-dimensional damping degree $(0<\xi<1)$ (Henclik, 2018a).

Usually, the fluctuation can be mathematically described as a pulse function which is expressed as Fourier series, e.g. The pulse delivery of the pump was taken as a sum of Fourier components (Henclik, 2018a). Our aim is mainly to explain the fluctuation of the vibration, and for simple calculation, we only consider the fundamental frequency in the pulsation. We assume that the time varying stiffness and damping $\Delta \alpha(t)$ and $\Delta \beta(t)$ are

$$
\Delta \alpha=\alpha_{0} \cos \left(\omega_{f} t\right) \quad \Delta \beta=\beta_{0} \cos \left(\omega_{f} t\right)
$$


where $\omega_{f}$ is the frequency related with the fluctuation. In the calculation, we suppose that $\omega_{f}=\omega_{0} / 5$. This assumption means that one fluctuation appears for five WH oscillations.

Substituting equation (4.1) into equation (3.4), the dynamic equation of the system under fluctuation conditions is obtained as

$$
m \ddot{y}+\beta_{0}\left[1+\cos \left(\omega_{f} t\right)\right] \dot{y}+\alpha_{0}\left[1+\cos \left(\omega_{f} t\right)\right] y=\rho v_{0} c S\left[1-\cos \left(k_{0} L\right) \cos \left(\omega_{0} t\right)\right]
$$

According to equation (4.2), the vibration displacement can be calculated. The numerical results are calculated by the Runge-Kutta method. The calculation flow chart is attached in Fig. 2. In the calculation, when the vibration displacement reaches the critical value, cavitation occurs. In the cavitation case, the pressure pulsation acting on the valve is vapor, and the vapor density $\rho_{v}$ replaces the density $\rho$ in the equation. Meanwhile, the time-varying coefficients $\Delta \alpha(t)$ and $\Delta \beta(t)$ are considered as zero. When the displacement recovers within the range of the critical value, WH pulsation acts on the valve section again. At the moment of pressure transition between the vapor and liquid, the system vibration is continuous.

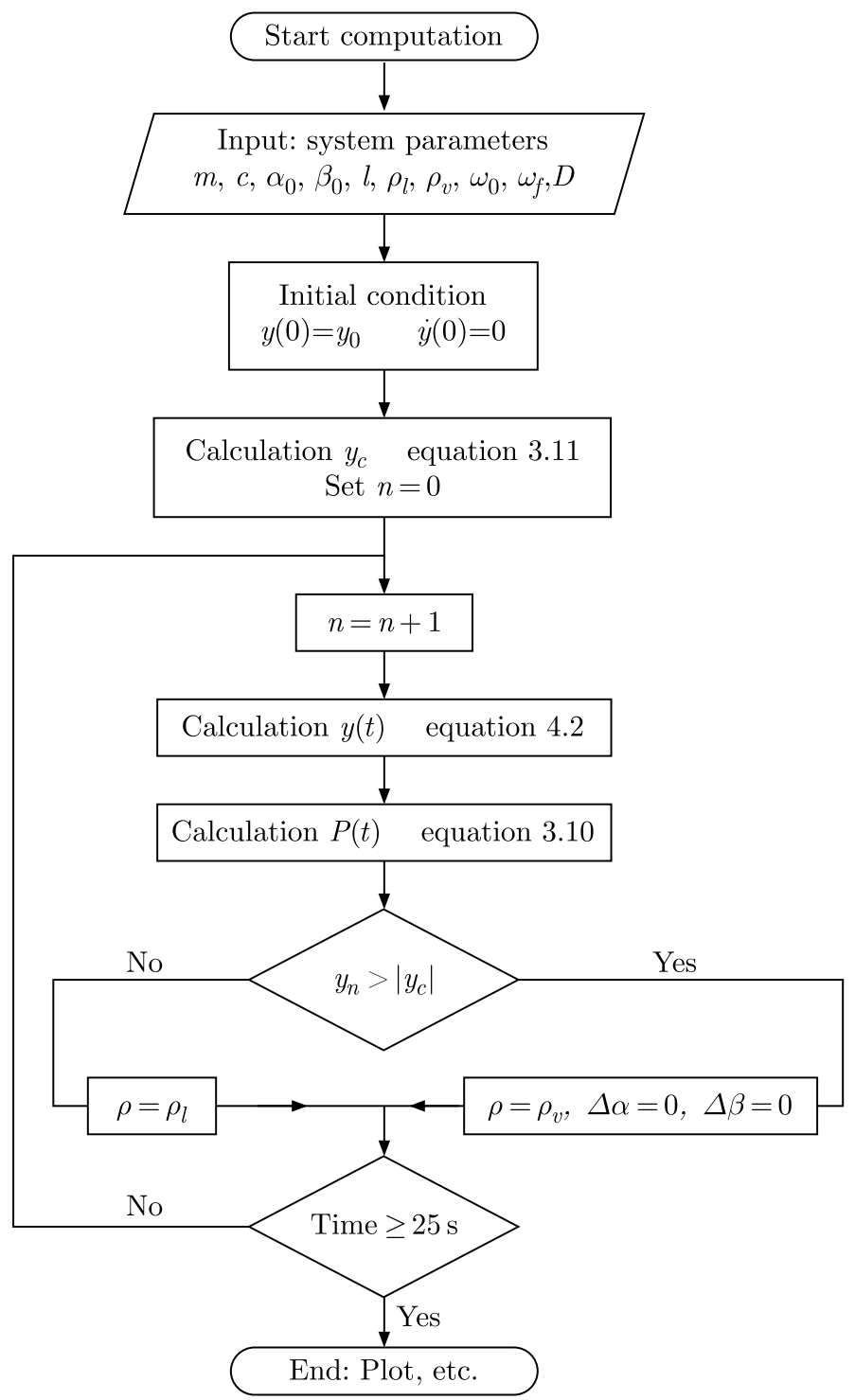

Fig. 2. Calculation flow chart 
Our test of the RPV system is designed for an aircraft fuel supply system. A detailed description of the experimental setup and parameters was given in (Miao et al., 2020), which can be referred to by interested readers. In the experiment, a computer executes the valve closing command automatically. The closing operation has a 5 seconds delay, i.e. after 5 seconds of the command, the valve starts closing. The valve can be closed within $10 \mathrm{~ms}$. When the fuel flowrate is $0.8 \mathrm{~m} / \mathrm{s}(56 \mathrm{~L} / \mathrm{min})$, the whole pipeline and valve system begin to vibrate violently and randomly as the valve is closing. At the same time, it is accompanied by an abnormal "click" sound. A transducer reads the pressure transients in the pipe (shown in Fig. 3). Subtracting the 5s delay, the violent oscillation lasts for about $15 \mathrm{~s}$ and then the system returns to a stable state. At the beginning of oscillation, the pressure rises rapidly from $0.3 \mathrm{MPa}$ to $2.6 \mathrm{MPa}$ within a short time. Then, the pressure amplitude decreases gradually, and finally the pressure returns to the preset stable state of $0.7 \mathrm{MPa}$. It is obvious that there are random, flickering and large amplitude fluctuations in the pressure oscillation.

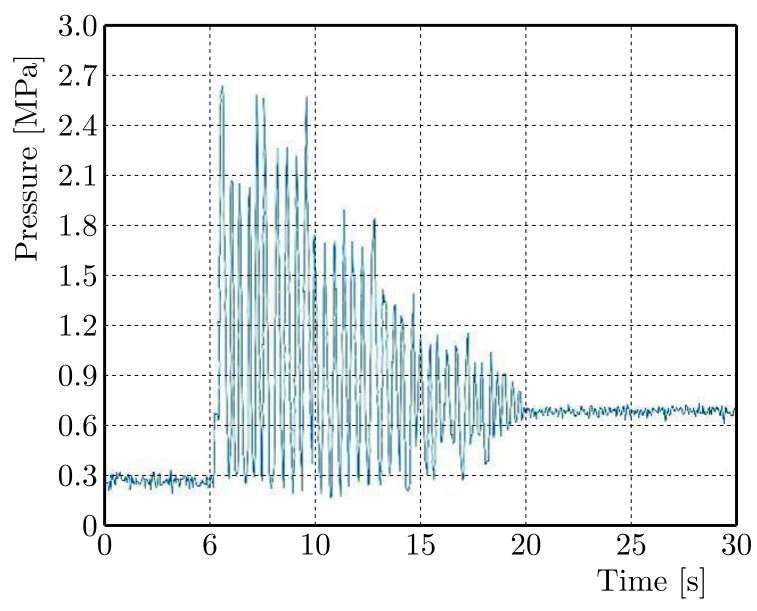

Fig. 3. Pressure transients recorded experimentally during the valve closing

(a)

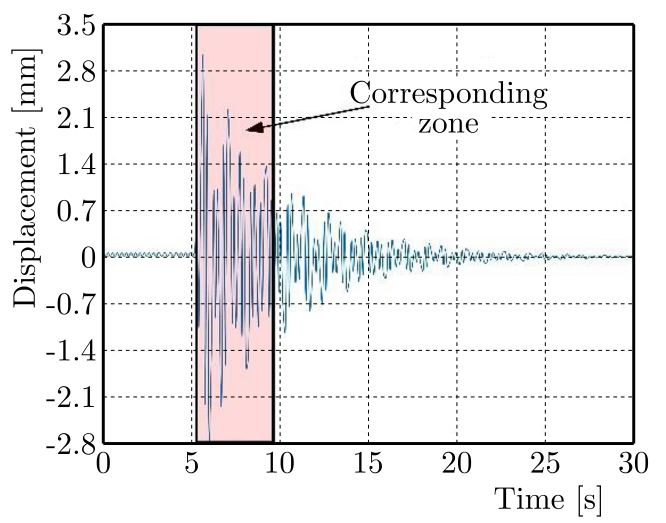

(b)

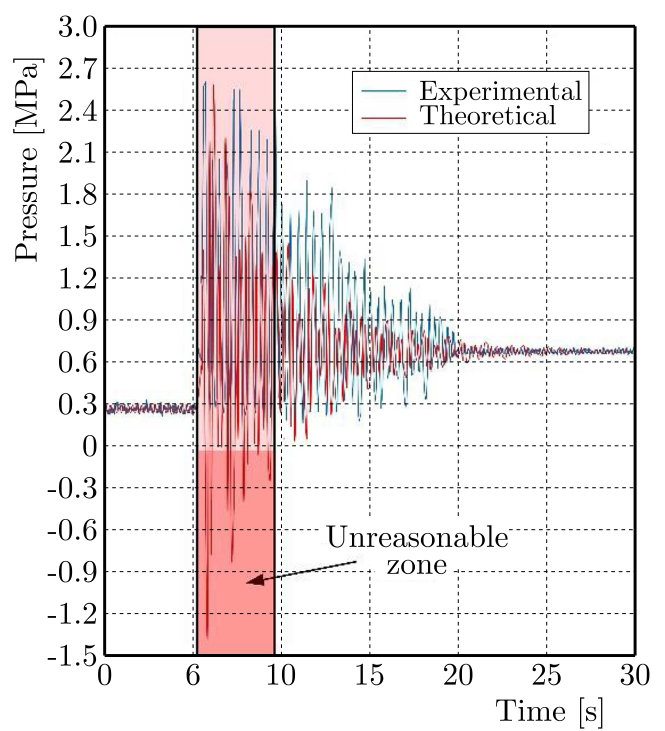

Fig. 4. Simulation transients of the vibration (a) displacement and pressure (b) based on the fluctuation model

Figure 4 depicts the simulated transients of the vibration displacement and pressure with the fluctuation model. As can be seen from Fig. 4a, the piping system vibrates when the valve is closed. As a whole, the vibration amplitude gradually decreases with time, and finally tends 
to be stable. However, the attenuation trend is not like the exponential and fluctuation in the transient process. Figure $4 \mathrm{~b}$ shows the liquid pressure transient at the valve. It is verified that the pressure vibrates when the valve is closed, and the pressure variation range is large. The pressure oscillation gradually decreases with time, and finally tends to be stable at $0.7 \mathrm{MPa}$. However, the most important fact is that the damping process is not completely exponential, and the fluctuation observed in the experiment as shown in Fig. 3 appears in the simulation prediction. The appearance of this abnormal click may be related to these fluctuations. Although the simulation based on the fluctuation model can explain the violent random vibration and abnormal sound observed in the experiment, the calculation results also have serious defects. As can be seen from Fig. 4b, there is a certain negative pressure in the simulation transient. Obviously, these pressure values are unreasonable and not in line with the reality. In order to solve this problem, a fluctuation model with flow cavitation is proposed. If the vibration amplitude of the pipeline system reaches the critical displacement, the flow cavitation should be considered. In this case, when the vapor column acts on the valve, the pressure is caused by vapor. Of course, if the vibration of the pipeline system is within the critical displacement range, the effect on the valve is $\mathrm{WH}$ pressure pulsation.

(a)

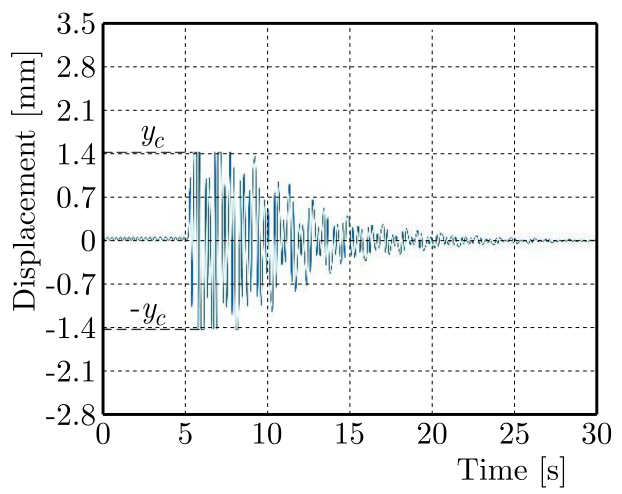

(b)

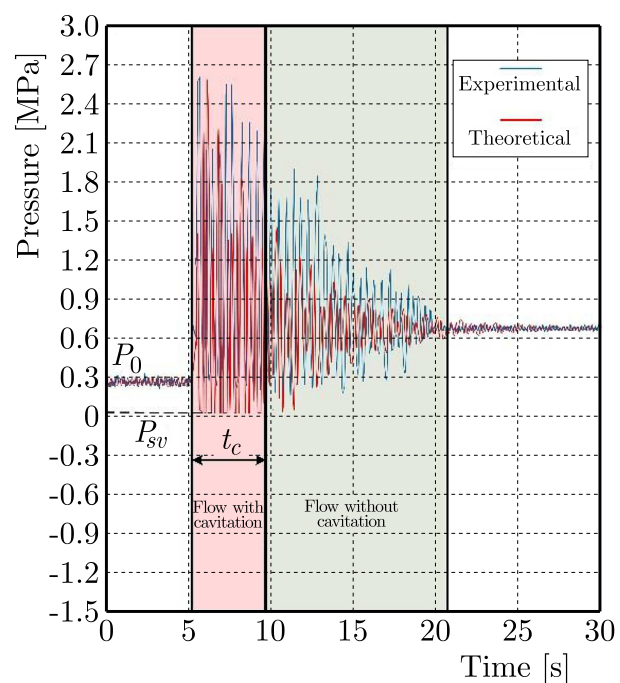

Fig. 5. Simulation transients of the vibration (a) displacement and (b) pressure based on the fluctuation with the flow cavitation model

Figure 5 depicts the simulated transients of vibration displacement and pressure based on the fluctuation model with flow cavitation. It can be clearly seen from Fig. 5a that the pipeline system vibrates when the valve is closed. As same as shown in Fig. 4a, from the overall trend, the vibration amplitude gradually decreases with time, and finally tends to be stable. The fluctuation also appears in the transient process. However, different from Fig. 4a, the vibration amplitude in Fig. 5a is limited in the range of critical displacement. Under our experimental conditions, according to equation (3.11), the critical displacement of vibration is $1.41 \mathrm{~mm}$. When the amplitude reaches the critical displacement, cavitation occurs and a vapor column is formed near the valve. In this case, the vapor pressure acts on the valve. Figure 5b shows transient simulation of liquid pressure at the valve. With the closing of the valve, the pressure vibrates, and the pressure oscillation gradually decreases with time, and finally tends to be stable at $0.7 \mathrm{MPa}$. There are fluctuations in the process. Compared with Fig. 4b, it is clear that the unreasonable pressure value in Fig. 5b disappears. Based on the fluctuation model with flow cavitation, when the vibration of pipeline system reaches the critical displacement, a vapor column is formed and the vapor pressure acts on the valve. In Fig. 5b, the maximum pressure is not at the first peak, but at the second peak. This may be due to the superposition of the 
pressure wave caused by the sudden closing of the valve and the appearance of the vapor column (Zarzycki and Urbanowicz, 2008). According to the definition of critical displacement and flow cavitation life time, the simulation in Fig. 5b shows that the cavitation life time is $4.48 \mathrm{~s}$. It can also be seen from Fig. 5b that the pressure transient can be divided into two parts, one is a cavitating flow, the other is a non cavitating flow. In the case of the cavitating flow, the pressure acting on the valve is composed of $\mathrm{WH}$ pressure and vapor pressure. The minimum pressure in this range can be approximately considered as saturated vapor. However, in the absence of cavitation, the pressure is completely caused by the flow. In order to quantitatively describe the agreement between the simulation and experiment, the average relative pressure peak error is defined (Zarzycki and Urbanowicz, 2008). If $p_{s i}$ and $p_{e i}$ are used to represent the amplitudes of the $i$-th pressure peak in the simulation and experiment, respectively, the average relative pressure peak error $\Delta p$ is expressed as $\Delta p=(1 / n) \sum_{i=1}^{n}\left|p_{s i}-p_{e i}\right| / p_{e i}$, where $n$ is the number of pressure peaks in statistics calculation. The error $\Delta p$ between our simulation and experiment is 0.022 , which shows the simulation result is in a good agreement with the experiment.

\section{Conclusions}

- It is observed that the RPV system vibrates violently when the valve is closed, accompanied by a series of abnormal "click" sounds. At the same time, there are large fluctuations in the detected pressure transient data. These fluctuations may lead to violent random vibration and abnormal sound.

- A fluctuation model with flow cavitation is proposed to simulate vibration of the RPV system. In this model, the system is equivalent to a spring and damper, and the time-varying coefficients of stiffness and damping are introduced to describe the vibration process. At the same time, if the system vibrates violently, the flow cavitation is considered. Due to flow cavitation, a vapor column is formed and the pressure acting on the valve is the vapor.

- A dynamic vibration equation of cavitating flow is established. In the equation, the periodic time-varying terms of stiffness and damping coefficients are used, and the exciting force acting on the valve takes into account both the WH pressure and the vapor pressure. According to the dynamic equation and the boundary condition of what the valve velocity is equal to, the flow velocity and the expression for the flow pressure at the valve is derived.

- The critical displacement of the valve section is defined. If the vibration amplitude of the system is within the critical displacement range, the pressure acting on the valve is water hammer. However, if the vibration amplitude reaches the critical displacement, the pressure acting on the valve is vapor. According to the flow pressure and pressure boundary conditions of the vapor column, the expression of the critical displacement is derived. Under our experimental conditions, the critical displacement of vibration is $1.41 \mathrm{~mm}$.

- Based on the fluctuation model and the fluctuation model with flow cavitation, the numerical results of the dynamic vibration equation of the RPV system are calculated by the Runge-Kutta method. The simulation processes of vibration, displacement and pressure are obtained, respectively.

- The simulations show that the result of the fluctuation model with flow cavitation is in a good agreement with the experiment data. The two simulations (based on the fluctuation model and the fluctuation model with flow cavitation) are compared. Although both of them show that the pressure in the pipeline vibrates violently, the unreasonable value in the result of the original fluctuation model disappears in the fluctuation model with flow cavitation. The simulation result of the new model is compared with the experimental data, and the average relative pressure peak error is 0.022 . 


\section{Funding}

This research was funded by National Natural Science Foundation of China, grant number (51975011, 51605009 and 51605010), the International Research Cooperation Seed Fund of Beijing University of Technology (No. 2021B24) and Beijing Postdoctoral Funding (2017-ZZ-036).

\section{References}

1. Adamkowski A., Henclik S., Janicki W., Lewandowski M., 2017, The influence of pipeline supports stiffness onto the water hammer run, European Journal of Mechanics B/Fluids, 61, 297-303

2. Covas D., Stoianov I.N., Mano J.F., Ramos H., Graham N., Maksimovic C., 2005, The dynamic effect of pipe-wall viscoelasticity in hydraulic transients. Part II - Model development, calibration and verification, Journal of Hydraulic Research, 43, 1, 56-70

3. Ferras D., Manso P.A., Schleiss A.J., Covas D., 2018, One-dimensional fluid-structure interaction models in pressurized fluid-filled pipes: A review, Applied Sciences, 8, 1844-1877

4. HenCliK S., 2015, A numerical approach to the standard model of water hammer with fluid-structure interaction, Journal of Theoretical and Applied Mechanics, 53, 3, 543-555

5. Henclik S., 2018a, Analytical solution and numerical study on water hammer in a pipeline closed with an elastically attached valve, Journal of Sound and Vibration, 417, 245-259

6. HencliK S., 2018b, Numerical modeling of water hammer with fluid-structure interaction in a pipeline with viscoelastic supports, Journal of Fluids and Structures, 76, 469-487

7. Keramat A., Tijsseling A.S., Hou Q., Ahmadi A., 2012, Fluid-structure interaction with pipe-wall viscoelasticity during water hammer, Journal of Fluids and Structures, 28, 434-455

8. Miao Y., Jiang Y., Qiu Z., Pan J., Wang L., Han Z., Zhang X., 2020, Vibration transients of reservoir-pipe-valve system caused by water hammer, Journal of Theoretical and Applied Mechanics, 58, 4, 1037-1048

9. Streeter V.L., 1969, Water hammer analysis, ASCE Journal of the Hydraulic Division, 95, 6, 1959-1972

10. TiJsseling A.S., 2003, Exact solution of linear hyperbolic four-equation system in axial liquid-pipe vibration, Journal of Fluids and Structures, 18, 2, 179-196

11. Urbanowicz K., 2017a, Analytical expressions for effective weighting functions used during simulations of water hammer, Journal of Theoretical and Applied Mechanics, 55, 3, 1029-1040

12. Urbanowicz K., 2017b, Modern modeling of water hammer, Polish Maritime Research, 24, 68-77

13. Urbanowicz K., Zarzycki Z., 2008, Transient caviting pipe flow: Computation models and methods, Task Quarterly, 12, 3, 159-172

14. URBAnOwicZ K., Zarzycki Z., 2012, New efficient approximation of weighting functions for simulations of unsteady friction losses in liquid pipe flow, Journal of Theoretical and Applied Mechanics, 50, 2, 487-508

15. URBAnowicz K, ZarZyCKi Z., 2015, Improved lumping friction model for liquid pipe flow, Journal of Theoretical and Applied Mechanics, 53, 2, 295-305

16. Urbanowicz K., Zarzycki Z., Kudźma S., 2012, Universal weighting function in modeling transient cavitating pipe flow, Journal of Theoretical and Applied Mechanics, 50, 4, 889-902

17. WAng Z.M., EAT TAN S.K., 1997, Coupled analysis of fluid transients and structural dynamic responses of a pipeline system, Journal of Hydraulic Research, 35, 1, 119-131

18. Wiggert D.C.,Tijsseling A.S., 2001, Fluid transients and fluid-structure interaction in flexible liquid-filled piping, Applied Mechanics Reviews, 54, 5, 455-481 
19. Wylie E.B., Streeter V.L., 1978, Fluid Transients, McGraw-Hill, New York

20. Wylie E.B., Streeter V.L., Suo L., 1993, Fluid Transients in Systems, Englewood Cliffs: NJ, USA

21. YAng K., Li Q.S., Zhang L., 2004, Longitudinal vibration analysis of multi-span liquid-filled pipelines with rigid constraints, Journal of Sound and Vibration, 273, 1-2, 125-147

22. Zanganeh R., Ahmadi A., Keramat A., 2015, Fluid-structure interaction with viscoelastic supports during waterhammer in a pipeline, Journal of Fluids and Structures, 54, 215-234

23. Zarzycki Z., Kudźma S., Kudźma Z., Stosiak M., 2007, Simulation of transient flow in a hydraulic system with a long liquid line, Journal of Theoretical and Applied Mechanics, 45, 4, 853-871

24. ZARZYCKI Z., KudŹma S., URBAnOwiCZ K., 2011, Improved method for simulating transients of turbulent pipe flow, Journal of Theoretical and Applied Mechanics, 49, 1, 135-158

25. ZARZYCKI Z., URBAnOWICZ K., 2008, The influence of hydraulic pressure conduits parameters on the course of unsteady flow with cavitation, Terotechnology, Scientific Problems of Machines Operation and Maintenance, 43, 1, 89-100

Manuscript received March 6, 2020; accepted for print June 22, 2021 